
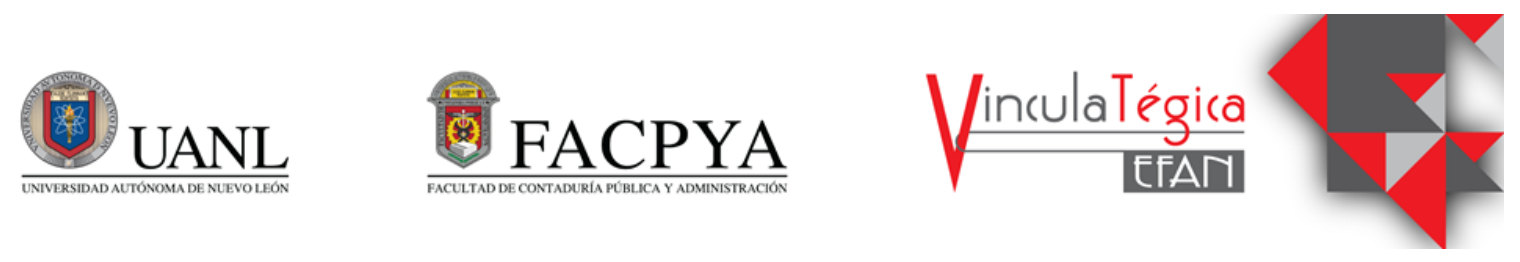

\title{
Estudio bibliométrico de la relación del Cambio Organizacional con el Aprendizaje Organizacional
}

\author{
Luis Alberto Bellon Álvarez ${ }^{1}$, Francisco Javier López Cerpa ${ }^{2}$ y Margarita Isabel Islas \\ Villanueva ${ }^{3}$ \\ ${ }^{1}$ Universidad de Guadalajara, luis_bellon2000@hotmail.com, Periférico Norte No 799, Núcleo \\ Universitario Los Belenes, C.P. 45100, Zapopan, Jalisco, México, 3334938148 \\ ${ }^{2}$ Universidad de Guadalajara, francisco.lcerpa@academicos.udg.mx, Periférico Norte $N^{\circ} 799$, Núcleo \\ Universitario Los Belenes, C.P. 45100, Zapopan, Jalisco, México, 3339685780 \\ Universidad de Guadalajara, margarita.islas@cucea.udg.mx, Periférico Norte $N^{\circ} 799$, Núcleo Universitario \\ Los Belenes, C.P. 45100, Zapopan, Jalisco, México, 3316674086
}

Información del artículo revisado por pares

Fecha de aceptación: junio-2021

Fecha de publicación en línea: diciembre-2021

DOI: https://doi.org/10.29105/vtga7.1-155

\begin{abstract}
Resumen
Abstract

Para este artículo se realizó un análisis bibliométrico de la For this article, a bibliometric analysis of the situación actual del estudio del impacto del aprendizaje current situation of the study of the impact of organizacional en un proceso de cambio organizacional. organizational learning in a process of El objetivo de esta investigación fue hacer un estudio que organizational change was carried out.

refleje las tendencias de investigación sobre la relación The objective of this research was: make a study entre el cambio organizacional y el aprendizaje that reflects the research trends on the relationship organizacional. Por lo que se efectuó una búsqueda de las between organizational change and organizational variables entre títulos, resúmenes, así como palabras clave, learning. Therefore, a search of the variables was de los documentos científicos obtenidos del repositorio carried out between titles, abstracts, and keywords, académico Web of Science. Llevando a cabo la búsqueda of the scientific documents obtained from the de las publicaciones a través de las palabras claves academic repository Web of Science. Carrying out elegidas, que fueron: "cambio organizacional" $y$ the search of the publications through the chosen "aprendizaje organizacional". Dichas palabras keywords, which were: "organizational change" determinaron el área de conocimiento, así como el área de and "organizational learning". These words acción del tema objeto de investigación. Dando como determined the area of knowledge, as well as the resultado de esta búsqueda un total de 217 documentos area of action of the subject under investigation. científicos. Los datos obtenidos fueron procesados con el Giving as a result of this search a total of 217 programa VOSviewer, mediante el método de recuento de scientific documents. The data obtained were coocurrencias, en función de las palabras claves indexadas. processed with the VOSviewer software, using the Dando como resultado cinco clústeres que muestran las co-occurrence count method, based on the indexed distintas perspectivas de cómo se ha abordado este tema. keywords. Resulting in five clusters that show the Este trabajo proporciona un panorama general del tema different perspectives of how this topic has been estudiado y puede ser útil para empresarios, investigadores approached. This work provides an overview of the y estudiosos del tema. Se sugiere efectuar una revisión más subject studied, and may be useful for profunda de la literatura sobre el tema y de las entrepreneurs, researchers and scholars of the investigaciones realizadas, para así poder identificar las subject. It is suggested to carry out a more in-depth
\end{abstract}


herramientas y áreas de aplicación que hay actualmente. Se review of the literature and the research carried out, puede concluir que los resultados de la investigación in order to identify the tools and areas of application muestran que hay un aumento en las aportaciones y that currently exist. It can be concluded that the publicaciones del tema, así como en su frecuencia de results of the research show that there is an increase publicación. in contributions and publications on the subject, as well as in its frequency of publication.

Palabras clave: Aprendizaje Organizacional, Cambio Organizacional, Organización Inteligente, Organización Keywords: Organizational Learning, que Aprende. Organizational Change, Intelligent Organizations, Learning Organization.

Códigos JEL:

M. Administración de empresas y economía JEL Codes: empresarial • Marketing C Contabilidad•Economía de M. Business Administration and Business personal

Economics $\bullet$ Marketing • Accounting • Personnel

M1 Administración de Empresas

M11 Gestión de la producción Economics

M12 Gestión del persona

Compensación a Ejecutivos

M14 Cultura corporativa • Diversidad

Responsabilidad social

\section{INTRODUCCIÓN}

El cambio es parte de nuestra realidad y está invariablemente presente. Hoy en día, una sucesión de variables converge e influyen en el desempeño de las empresas. Un ejemplo de todo esto se puede ver en cómo la pandemia producida por el Coronavirus, ha producido un notorio cambio en la manera de trabajar de las organizaciones, así como en los hábitos de consumo. Por tal razón, realizar un estudio sobre el proceso de cambio en las empresas es elemental, ya que, como se sabe es necesario tener una correcta gestión empresarial. Como se señaló, el mundo continuamente está cambiando, siendo los mercados y las empresas parte de esos cambios. Por ello, se debe analizar el proceso de cambio, para entender cabalmente cómo es la dinámica de dicho proceso de cambio, así como los aspectos o factores que lo originan. A su vez, resulta de gran utilidad saber cuáles son los factores que influyen en un proceso de cambio en las organizaciones.

Además, hay que señalar que el mundo ha estado cambiando desde fines del siglo $\mathrm{XX}$, a un ritmo muy vertiginoso, lo que ocasionó que no fuéramos consientes de dichos cambios, y no nos detuviéramos a
M1 Business Administration

M11 Production Management

M12 Personnel Management - Executives;

- Executive Compensation

M14 Corporate Culture • Diversity • Social Responsibility

pensar en las consecuencias que tales cambios traerían al mundo. Hoy en día, nos damos cuenta de que las habilidades y prácticas que conocíamos antes se han vuelto obsoletas.

Por su parte, el enfoque de la perspectiva de estudio del Aprendizaje Organizacional, muestra que los cambios que se presentan son una clase de alteración que exhibe el negocio. Una organización que aprende favorece que la gente se adecúe al cambio, ya que reaccionan con más prontitud ante los cambios $y$, a su vez, pueden anticiparlos. De esta forma, los conocimientos se han vuelto el principal insumo en el ámbito empresarial.

\section{MARCO TEÓRICO}

Cómo se lleva a cabo un cambio organizacional implica mucho trabajo, ya que cualquier cambio debe encarar un conjunto de problemas que obstaculizan su implementación. De ahí la importancia de realizar este tipo de investigaciones. Así como el mundo está en constante cambio, las empresas también cambian por las razones antes mencionadas. Por lo tanto, se debe entender cómo cambian las empresas y la razón de estos cambios.

Para implementar una estrategia que 
haga a una organización más eficiente y competente, se debe contar con una orientación integral hacia el aprendizaje organizacional, acorde a los desafíos que demanda el mercado; por lo que la inteligencia organizacional tiene un papel fundamental en el desarrollo de organizaciones que aprenden.

Entre los autores que examinan el cambio organizacional desde el punto de vista de la corriente de estudio del Aprendizaje Organizacional, están: Al Kasasbeh, Al Kasasbeh, \& AL Faouri, (2016); Bahrami, Kiani, Montazeralfaraj, Zadeh, \& Zadeh, (2016); Erçetin, \& Kayman, (2014); Holba, Bahr, Birx y Fischler (2019); Keskin, y Balak, (2020); Ravichandran, \& Mishra, (2018); Šneiderienè, Vaitiekus, y Vaitiekienè, (2020); Tortorella, \& Cawley-Vergara, Garza-Reyes, \& Sawhney, (2020); Walker, C. (2014); que fueron analizados en este estudio.

El aprendizaje organizacional contribuye a que las empresas permanezcan en el mercado, desarrollen nuevas habilidades y capacidades, y les ayuda a hacer frente al cambio y aprovechar nuevas oportunidades. Al respecto, Limwichitr, Broady-Preston y Ellis (2015), abordan la importancia de explorar el cambio de cultura organizacional como un aspecto que favorece el desarrollo de una Organización que Aprende. En tanto que Pole, Madsen y Dishman (2000), indican que el aprendizaje organizacional y la decisión junto con la inteligencia emocional, que viene a ser una subdimensión de la inteligencia organizacional, son una parte importante de la participación en el proceso de recepción, lo que le da a la empresa la capacidad de gestionar el cambio y mejorar su rendimiento.

La competitividad organizacional comprende el concepto de organización que aprende. La organización que aprende es aquella organización donde todo el personal participa en identificar y resolver problemas, lo que hace que la organización incremente constantemente su capacidad para crecer, aprender y alcanzar su propósito. El principio organizativo de una organización que aprende no viene a ser su eficacia o eficiencia, sino la capacidad para solucionar problemas y su competitividad. (Ugoani, 2016).

La teoría del aprendizaje organizacional considera el desarrollo, aprovechamiento, conservación, transmisión y aplicación de los conocimientos dentro de la empresa (Bahrami, Kiani, Montazeralfaraj, Zadeh y Zadeh, 2016). También permite que se renueve la empresa para que pueda estar al día con respecto al entorno de trabajo. A su vez, conservar y mejorar el desempeño organizacional en entornos en constante cambio se relaciona con habilidades de aprendizaje organizacional (Virany, Tushman y Romanelli, 1992). Además, el aprendizaje organizacional es un elemento esencial de la inteligencia organizacional. (Keskin y Balak, 2020). El hecho de que una empresa contenga sistemas para pronosticar, seguir, responder y adecuar los cambios, son aspectos tanto del aprendizaje organizacional como de la inteligencia organizacional (Liebowitz, 2000).

El aprendizaje organizacional impacta positivamente en el rendimiento del personal. Las organizaciones con alto nivel de inteligencia organizacional, aprendizaje y desempeño; trabajan eficazmente, y son capaces de interpretar señales; y actúan sabiamente ante distintos acontecimientos y aprenden de sus nuevas experiencias.

Por su parte, Tortorella, Vergara, Garza-Reyes y Sahney (2020), indican que el aprendizaje organizacional puede verse como un proceso de mejora, que se basa en un mayor entendimiento $\mathrm{y}$ en un conocimiento directamente relacionado con el ambiente empresarial y su cultura organizacional. En tanto que Salge y Vera (2012), establecen que las organizaciones que aprenden entienden la importancia del aprendizaje e incrementan la habilidad de convertir los nuevos conocimientos en mejoras tangibles.

Por otra parte, el principio del aprendizaje continuo indica que el aprendizaje se llevará a cabo de tres formas: adaptándose, produciéndose y transformándose con el aprendizaje continuo. El aprendizaje está influenciado por el medio ambiente, y libera el conocimiento; y esta relación es controlada por la disposición de la organización para aprender. La preparación para el aprendizaje es una función de la permeabilidad de los límites de la empresa. Las organizaciones aprenden y se desarrollan por medio de sus 
propias experiencias y las del personal con que laboran, así como de otras entidades y agrupaciones (London y Sessa, 2006). Una cultura que desarrolle un aprendizaje permanente basado en el aprendizaje continuo, espera que las empresas solucionen los conflictos siguiendo una ruta de acción que sea la más adecuada. (Keskin y Balak, 2020).

Walker (2014), menciona varias características propias de las organizaciones que aprenden: colaboración, formulación de estrategias, inversión en el futuro, monitoreo ambiental, enfoque en identificar problemas y su solución, análisis y aplicación de grandes cantidades de información, respuesta y anticipación a cambios internos y externos.

El aprendizaje organizacional ha sido analizado por diversos investigadores, aunque muchos se centraron en habilidades y calificaciones; y sólo unos cuantos se enfocaron en los hábitos y comportamientos que generan un cambio reflexivo tanto en la persona como en la organización. (Ravichandran y Mishra, 2017). Así pues, el concepto de organización que aprende involucra cambios en los conocimientos, las prácticas y los puntos de vista de los integrantes de una organización. En síntesis, una misión clara y amplia permite a los trabajadores actualizar sus conocimientos y habilidades para así conseguir los resultados esperados. La organización que aprende tiene una ventaja competitiva en los nuevos mercados emergentes debido al hecho de que las aptitudes y las competencias coexisten. Tienen habilidades superiores para solucionar problemas conforme se relacionen y se involucren efectivamente, lo que se requiere en los principales puestos en las empresas.

Por otra parte, hay que indicar que el aprendizaje organizacional es un elemento primordial de la inteligencia organizacional. La inteligencia organizacional de una empresa se basa en la habilidad de esa compañía para aprender y adaptarse a los cambios que se presenten. Cabe señalar que aun cuando las empresas sean capaces de aprender y adaptarse a los cambios, esto no garantiza que se incremente la inteligencia en la organización.

Según Walker (2014), el conocimiento, el aprendizaje y la innovación son los factores fundamentales para asegurar el éxito de una empresa moderna. Pero, hay que mencionar que la adquisición, el uso y el reconocimiento de conocimientos dependen en gran medida de la cultura de la organización.

El aprendizaje organizacional se logra a través de obtener nuevos conocimientos, distribuir la información, saber cómo interpretar esa información, así como la memoria organizacional de la empresa. Por lo tanto, la organización que aprende debe asegurarse de que se tengan estos elementos: pensamiento sistémico, manejo de modelos mentales, desarrollo de una visión compartida, dominio personal y aprendizaje en equipo. Se puede afirmar que hay un vínculo entre una organización que aprende y la existencia de una mayor capacidad de innovación organizacional, mayor productividad y una ventaja competitiva. Hay que recalcar que para lograr el éxito se requiere intercambiar conocimientos, comunicar y aprender, aclarar y cambiar las prácticas existentes.

Abordar la teoría del liderazgo resulta algo muy basto, y en lo que se refiere a su estudio dentro del contexto de una organización que aprende, se puede mencionar que, en este caso, los sistemas de liderazgo transaccional y transformacional son muy patentes. Las organizaciones que aprenden necesitan contar con personal informado, motivado y que estén seguros de sí mismos y de que puedan conseguir nuevos conocimientos y tomar decisiones sobre cómo conseguir y utilizar dichos conocimientos. Por su parte, los enfoques del liderazgo compartido e inclusivo, son fundamentales para asegurarse de lograr un cambio organizacional que sea sostenible mediante la aplicación del aprendizaje organizacional. Existen 3 dimensiones sociales que contribuyen a desarrollar una organización que aprende: apertura a considerar distintos puntos de vista, proporcionar seguridad psicológica y el involucramiento en el proceso de toma de decisiones. La relación entre el aprendizaje organizacional y el liderazgo se puede analizar, mediante la cultura organizacional, los valores personales y colectivos de la organización, así como la colaboración, la confianza y la honestidad. La 
posibilidad de que surja y se exprese el liderazgo en una organización que aprende se relaciona con distintas variables: el líder, las circunstancias que ocasionan los cambios en la organización y los seguidores. Así pues, la teoría del liderazgo es muy amplia y variada, pero en el contexto de una organización que aprende, los tipos de liderazgo transaccional y transformacional, empoderador, colectivo y positivo son de gran importancia. (Šneiderienè, Vaitiekus y Vaitiekienè, 2020).

Las organizaciones inteligentes se caracterizan por trabajar incesantemente y ser capaces de adaptarse a ambientes impredecibles. Las organizaciones inteligentes también saben lidiar con la incertidumbre que les rodea aprendiendo a adecuarse a la complejidad dinámica que les asedia (Schwaninger, 2019). De esta forma, la inteligencia organizacional muestra organizaciones inteligentes que aprenden a gestionar la información de manera sabia. (Rahdarpour y Sheykhi, 2016).

Para poder ayudar acertadamente a sus organizaciones a manejar el cambio, los directivos por principio de cuentas deben ser reflexivos y saber gestionar sus propias emociones de preocupación e incertidumbre. Posteriormente, deben ser conscientes de las reacciones emocionales de otros integrantes de la empresa y ayudar a esas personas a encarar dichas reacciones. Como parte de ese proceso de hacer frente adecuadamente a un cambio masivo, otros miembros de la compañía tienen que participar activamente en el seguimiento y gestión de sus reacciones emocionales, así como las de los demás. (Ugoani, 2016).

Aceptar la incertidumbre en las organizaciones inteligentes conlleva el ser conscientes de que el futuro es imprevisible. Los integrantes de una organización inteligente deben saber cómo enfrentar a la incertidumbre y de qué forma incluirla dentro de los procesos de reflexión de la empresa. Así pues, la incertidumbre en una organización inteligente se comprende, se transmite y se gestiona. (Keskin y Balak, 2020).

Conforme el conocimiento se torna un activo en términos de competitividad, los mecanismos de aprendizaje, desaprendizaje y desarrollo de competencias se vuelven rasgos muy valiosos. (Argyris 2010).

Las organizaciones inteligentes desarrollan su base de conocimientos por medio de procesos de aprendizaje continuo, ayudados por la realización de diversos diálogos dentro de la organización. De esta forma, la comunicación y la interacción son básicas para poder efectuar cualquier proceso de gestión de cambio organizacional. Sin esto, el cambio no se podría llevar a cabo o sería irrelevante. Por lo que la capacidad para poder gestionar competencias se halla en el núcleo de cualquier organización inteligente (Fernández y Rainey 2006; Fernández y Pitts 2007). Por otra parte, dentro de la inteligencia organizacional, un aspecto primordial es la organización, sus características y sus funciones, como parte del proceso de transformación. (Ugoani, 2016).

El aprendizaje de acción suministra la base teórica y conceptual pragmática para esta narrativa y su utilización contribuye a que la empresa se vuelva una organización que aprende (Marquardt, 2000; Senge, 2006). Adicionalmente, el aprendizaje activo aporta equilibrio a la organización. (Cunliffe y Easterby-Smith, 2004; Rigg y Trehan, 2004). El aprendizaje activo viene a ser la metodología más acertada para analizar el trabajo organizacional en las empresas. Por lo que hay que tomar en cuenta en cada paso, qué es lo que se busca hacer, cómo se planea esto y cómo medirlo, para que así se logre que prospere la empresa. Conceptualmente hablando resulta ser un sistema iterativo de aprendizaje constructivista y de análisis de eventos, teniendo siempre en la mira la mejora continua de la empresa. (Coghlan, 2013; Marquardt, 2000; Revans, 1998).

Las organizaciones inteligentes con un alto nivel de aprendizaje, rendimiento e inteligencia organizacional, son capaces de operar eficazmente en escenarios complejos; tienen la habilidad de adquirir y comprender la información sobre los objetivos del negocio. Además, también son capaces de descifrar las señales que se presentan en su entorno, actuar con sabiduría ante distintas circunstancias y aprender de sus experiencias (Al Kasasbeh, Al Kasasbeh y Al Faouri, 2016).

Para Holba, Bahr, Birx y Fischler 
(2019), la integración es un modelo organizacional y de aprendizaje, consideran que determinar la expectativa sobre la importancia de volverse una organización que aprende es fundamental para lograr un cambio organizacional exitoso dentro de todo el sistema. Los sistemas pueden ser complejos y dinámicos; no puede asumirse que sean estáticos y sin emociones; ya que las personas son parte de los sistemas, todo el tiempo hay retos, cambios y amenazas que están en juego, y que llevan a un entorno dinámico que no siempre es seguro o predecible.

Aplicar la integración como metodología para lograr un cambio organizacional requiere de aprendizaje. El escrutinio del flujo de trabajo, el cambio de modelos mentales que han sido parte de identidades individuales, estimular a la gente a que no únicamente mejoren sus habilidades y capacidades, sino también a que sean más conscientes de cómo se desempeñan y quiénes son los que están trabajando, son rasgos fundamentales de la flexibilidad y la adaptabilidad en un ambiente laboral. Por lo que liderar el cambio organizacional mediante la integración conlleva que la empresa se vuelva una organización que aprende. Es importante tener un buen entendimiento del pensamiento sistémico y reconocer el papel de las interrelaciones y la interdependencia dentro de la cultura organizacional. (Holba, Bahr, Birx y Fischler, 2019),

Así pues, hay que señalar que, las organizaciones inteligentes y que aprenden pueden permanecer mucho tiempo ya que saben cómo adaptarse, renovarse, apresurar y aprender a cambiar rápidamente y de forma sencilla. (Erçetin y Caimán, 2014).

En conclusión, se puede afirmar que la gran rapidez de los cambios tanto: económicos como sociales, y tecnológicos que afrontan las empresas han influido considerablemente en el aprendizaje que tienen las organizaciones sobre sus mercados. Ya no funciona que las organizaciones reaccionen a dichos cambios, deben adelantárseles. No sirve conservar la posición actual, hay que alcanzar la delantera en el desarrollo, lo cual es un requerimiento para las empresas que son capaces de efectuar un proceso continuo de cambio en sus organizaciones. Aunque hay que señalar que la capacidad para cambiar no se compra, la organización tiene que aprenderla por su cuenta. Al abordar un cambio organizacional, se puede determinar que una firma empresarial ha cambiado cuando sus trabajadores se comportan de manera diferente. La gente es la que debe crear y cambiar a una organización por medio del aprendizaje, lo cual inicia con los directivos, quienes juegan un rol primordial en la organización.

\section{MÉTODO}

Este trabajo tiene 2 faces: 1.- una teórica, donde se investiga la parte teórica del tema; 2.- Un estudio bibliométrico sobre la relación del cambio organizacional y el aprendizaje organizacional.

Esta investigación se efectuó usando el método del análisis bibliométrico, que consiste en examinar el contenido semántico de la bibliografía del tema a estudiar, para así poder hacer inferencias de las coincidencias en cuerpos de texto, palabras clave, autores, entre otros (van Raan, 2019).

La aplicación del análisis bibliométrico permitió en esta investigación, identificar los tópicos más importantes en los trabajos que se encuentran en la base de datos académica Web of Science (WOS), la cual se consultó debido a que de acuerdo con Kulkarni et al (2009) y Bar-Ilan (2008), es una de las bases de datos más usadas para efectuar análisis bibliométricos (Olczyk, 2016). Para realizar la búsqueda en el repositorio académico Web of Science, las palabras clave que se utilizaron fueron: "cambio organizacional" Y "aprendizaje organizacional". Por lo que, para efectuar la búsqueda en la base de datos, se refino dicha búsqueda mediante distintos criterios, la búsqueda se hizo en los siguientes campos de las publicaciones: Título, palabras clave y resumen. De esta forma, para la búsqueda general se usaron los criterios: "Organizational Change" y "Organizational Learning", esto, con el propósito de aportar un marco sobre el estado existente en el momento de la presente investigación sobre los enfoques de trabajo de esos tópicos. Por otra parte, también se usó el software 
VosViewer para mostrar los mapas con las conexiones que se dieron entre las publicaciones encontradas.

\section{RESULTADOS}

El repositorio académico Web of Science muestra al 18 de abril de 2021 un total de 217 documentos científicos (194 artículos, 7 accesos anticipados, 6 materiales editoriales, 6 capítulos de libro, 1 reseña de libro, 1 resumen de reunión, 10 artículos de actas, 1 libro y 14 reseñas) que cuentan con los términos de los tópicos bajo estudio, ya sea en el título, el resumen o como palabra clave. La base de datos Web of Science ofrece la posibilidad de descargar los registros bibliográficos de los patrones analizados en varios formatos, que ofrecen la posibilidad de poder llevar a cabo un análisis en distintas plataformas. Para analizar la información conseguida para esta investigación se empleó el software VOSviewer, que permite visualizar las redes de co-ocurrencia de la información obtenida de los registros de la bibliografía. (van Eck y Waltman, 2010).

Con respecto al estudio bibliométrico, primeramente, los resultados obtenidos muestran los análisis propios que genera la base de datos Web of Science.

Figura 1. Producción científica sobre Cambio Organizacional y Aprendizaje Organizacional en Web of Science

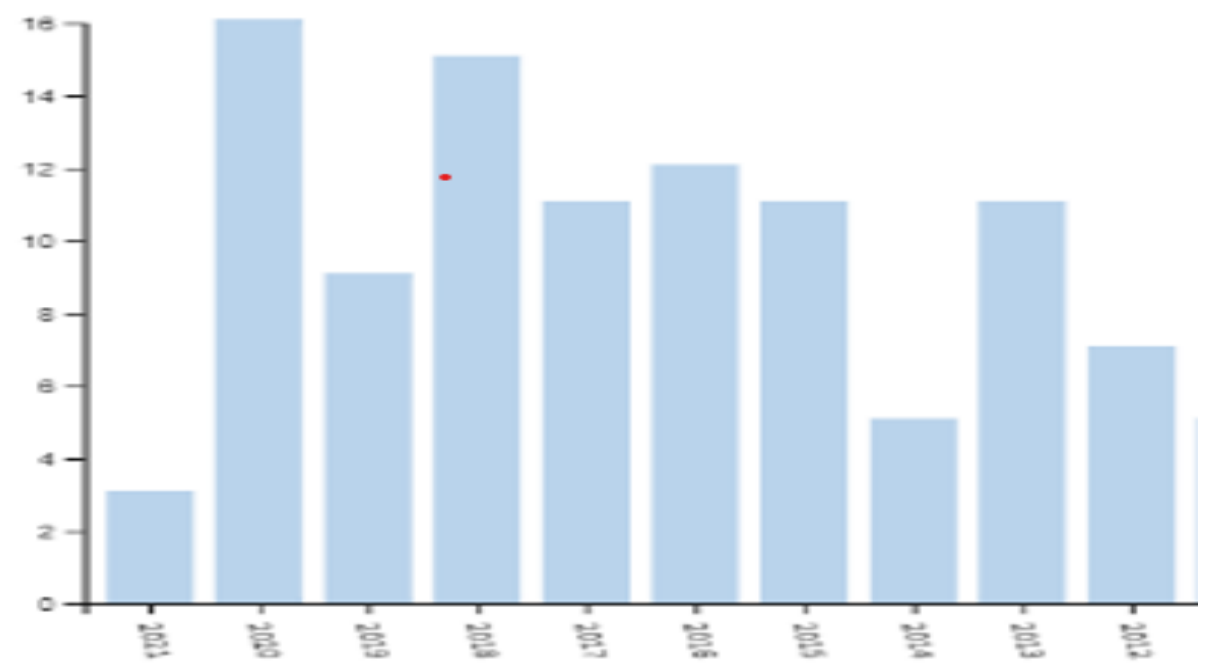

Fuente: Elaboración propia con base en los estadísticos de Web of Science

En la búsqueda de artículos que abordan la temática de la relación entre el cambio organizacional y el aprendizaje organizacional, se hallaron 217 documentos en la base de datos de Web of Science con una producción desde 1997 hasta 2021.
Como lo muestra la figura 1 , se dio un aumento significativo en publicación de artículos en el año 2018 teniendo un incremento en el año 2020, aunque tuvo una reducción significativa en el 2019.

Figura 2. Los 5 principales países de producción científica sobre Cambio Organizacional y Aprendizaje Organizacional 


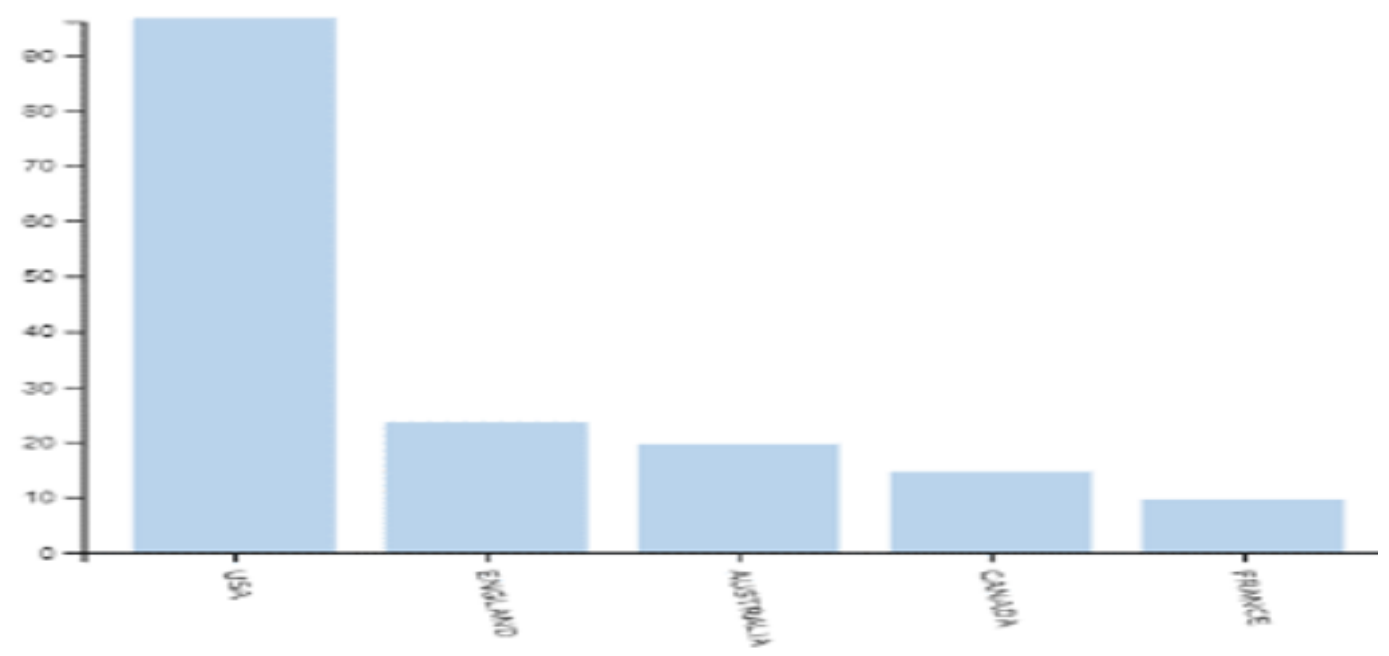

Fuente: Elaboración propia con base en los estadísticos de Web of Science

Los Estados Unidos lideran la producción científica sobre la relación entre el Cambio Organizacional y el Aprendizaje Organizacional, le siguen muy distantemente Inglaterra, Australia, Canadá, Francia y Alemania entre los países más productivos.

Por otra parte, los principales artículos, tomando como referencia para dicha clasificación el número de veces que fueron citados. El artículo con más citas es "Organizational Learning: The Contributing Processes And The Literatures" que tiene 3558 citas, y fue publicado por la revista Organization Science en 1991, del autor George P. Huber, quien afirma que la memoria organizacional requiere de una investigación sistemática, en especial de aquellos cuyas preocupaciones especiales son mejorar el aprendizaje organizacional y el proceso de toma de decisiones.

Como el segundo artículo más citado está: "Capabilities, cognition, and inertia: Evidence from digital imaging" con 1107 citas, de los autores Tripsas \& Gavetti, publicado en el año 2000 en la revista Strategic Management Journal, este trabajo señala que hay evidencia empírica de que las empresas frecuentemente tienen problemas para adaptarse a cambios radicales, también habla de las fuerzas inerciales asociadas con la naturaleza local de los procesos de aprendizaje, y establece que no se ha dado la debida atención teórica a entender de qué forma la cognición gerencial afecta la inteligencia adaptativa de las empresas.

El tercer artículo más citado con 474 citas es de los autores Zollo \& Singh, publicado en el 2004 en la revista Strategic Management Journal y lleva por título "Deliberate learning in corporate acquisitions: Post-acquisition strategies and integration capability in US bank mergers", en éste se indica que se puede aprender a gestionar el proceso de integración posterior a la adquisición acumulando la experiencia de adquisición y codificándola en manuales, sistemas y otras herramientas de adquisición; también indica que hay implicaciones para la teoría del aprendizaje organizacional y para el enfoque basado en el conocimiento para la investigación de estrategias corporativas. (tabla 1).

Tabla 1. Artículos más citados sobre cambio organizacional y estructura organizacional en Web of Science

\begin{tabular}{|l|l|l|l|}
\hline Autor & Título del artículo & Año & $\begin{array}{l}\text { Número } \\
\text { de citas }\end{array}$ \\
\hline $\begin{array}{l}\text { Huber, } \\
\text { George P. }\end{array}$ & $\begin{array}{l}\text { Organizational Learning: The Contributing Processes And } \\
\text { The Literatures }\end{array}$ & 1991 & 3558 \\
\hline $\begin{array}{l}\text { Tripsas, M; } \\
\text { Gavetti, G }\end{array}$ & $\begin{array}{l}\text { Capabilities, cognition, and inertia: Evidence from digital } \\
\text { imaging }\end{array}$ & 2000 & 1107 \\
\hline
\end{tabular}




\begin{tabular}{|l|l|l|l|}
\hline Zollo, M; & $\begin{array}{l}\text { Deliberate learning in corporate acquisitions: Post-acquisition } \\
\text { singh, H }\end{array}$ & 2004 & 474 \\
\hline
\end{tabular}

Fuente: Elaboración propia con base en los estadísticos de Web of Science

Como parte del estudio bibliométrico, también se analizaron las Categorías de investigación incluidas en el repositorio académico de Web of Science en las que fueron registradas las distintas publicaciones, la tabla 2 muestra los datos obtenidos de la base de datos Web of Science, siendo el área de Management la de mayor cantidad de artículos, seguido de la de Business, y luego de Information Science Libraryscience.

Tabla 2. Publicaciones por área disciplinar de las bases de datos Web of Science.

\begin{tabular}{|c|c|}
\hline Categorías de Investigación & Número de Artículos \\
\hline MANAGEMENT & 127 \\
\hline BUSINESS & 44 \\
\hline INFORMATION SCIENCE LIBRARYSCIENCE & 15 \\
\hline EDUCATION EDUCATIONAL RESEARCH & 14 \\
\hline HEALTH POLICY SERVICES & 13 \\
\hline
\end{tabular}

Fuente: Elaboración propia con los resultados obtenidos de Web of Science

Por otra parte, siguiendo con los resultados; hay que señalar que para efectuar el análisis se procesaron los 217 documentos en el programa VOSviewer, el cual generó los mapas basados en los datos bibliográficos, y se procedió a determinar los criterios de creación de los mapas.

En cuanto al estudio de la relación entre el cambio organizacional y el aprendizaje organizacional se halló que existen 4 clústeres de investigación el primero de ellos lo conforman Australia, Alemania, Inglaterra, y Suecia; el segundo clúster lo integran: Francia, Holanda, España y Estados Unidos de América, el tercer clúster está integrado por Canadá e Italia; y en el cuarto clúster están Dinamarca y Noruega. (Figura 3).

Figura 3. Mapa de densidad por países

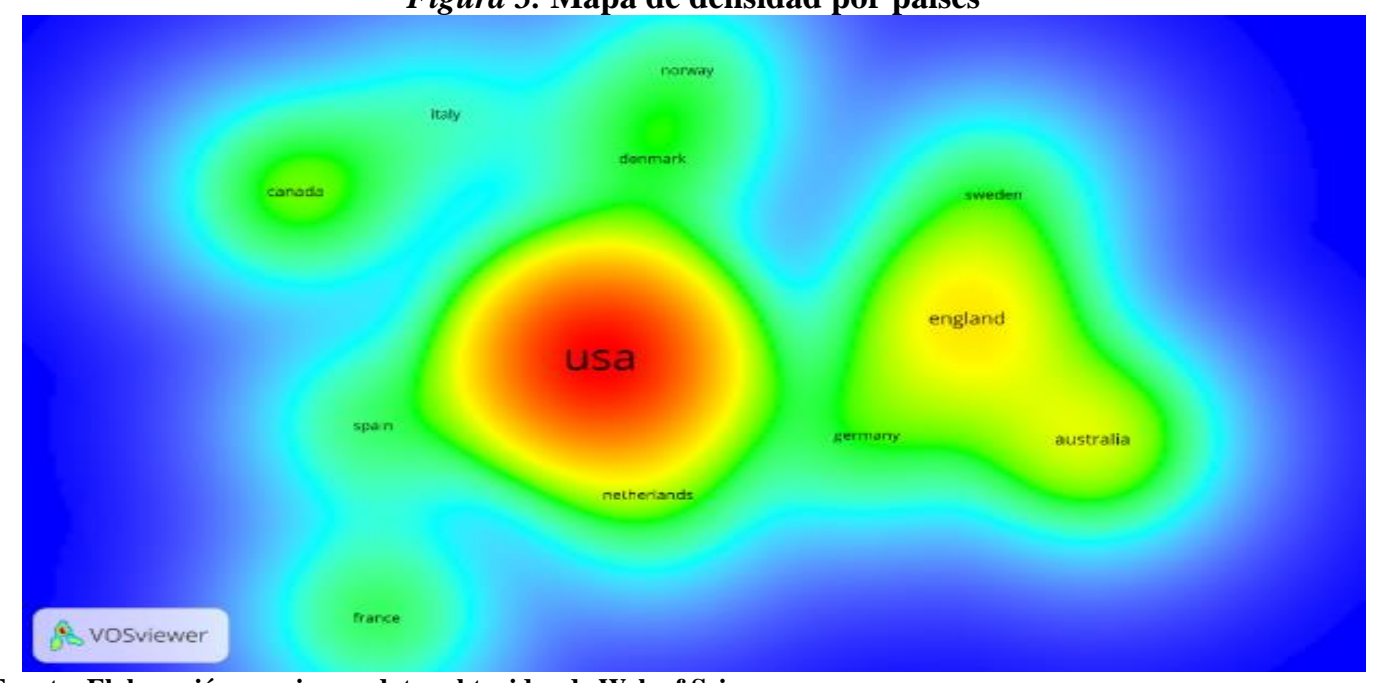

Fuente: Elaboración propia con datos obtenidos de Web of Science

Siguiendo con el análisis de los documentos. Se optó para este proyecto generar mapas bibliométricos que se fundamenten en co-ocurrencias, lo cual se determina de acuerdo al número de veces que aparecen las palabras claves en los documentos que se descargaron de la búsqueda que se hizo en la base de datos Web of Science, y dichos datos se usaron para crear una red. Se escogieron las palabras 
claves indexadas al correr el programa del VOSviewer, dichas palabras claves son seleccionadas de forma automática por la propia base de datos, cabe mencionar que es preferible usar esta clase de palabras claves ya que de esta forma se cuenta con un panorama más claro y completo de cómo es la dinámica que se presenta en el campo de estudio que se esté analizando, ya que así no se circunscriben únicamente a las palabras claves indicadas por el autor. (Gálvez, 2016).

Para esta investigación se determinó hacer un análisis y procedimiento de recuento de co-ocurrencias tomando como base las palabras claves indexadas, eligiendo un número mínimo de co-ocurrencias en las palabras claves $\geq 5$, la elección de este umbral se determinó tomando en cuenta que el resultado de co-ocurrencias fue de 1003 palabras claves por lo que si se optaba por un menor umbral el mapa tendría demasiadas coincidencias lo que impediría hacer un adecuado análisis, ya que se superpondrían las etiquetas unas con otras.

Tabla 3. Relación de palabras clave por importancia de apariciones y fuerza de enlace

\begin{tabular}{|l|l|l|}
\hline Palabra clave (Keyword) & $\begin{array}{l}\text { Ocurrencias } \\
\text { (Ocurrences) }\end{array}$ & $\begin{array}{l}\text { Fuerza total del enlace } \\
\text { (Total Link Strength) }\end{array}$ \\
\hline Organizational learning & 107 & 372 \\
\hline Organizational change & 91 & 288 \\
\hline Performance & 40 & 200 \\
\hline Innovation & 32 & 169 \\
\hline Model & 35 & 168 \\
\hline Organizational-change & 40 & 166 \\
\hline Management & 39 & 165 \\
\hline Knowledge & 20 & 99 \\
\hline Culture & 19 & 70 \\
\hline Leadership & 19 & 69 \\
\hline Implementation & 16 & 68 \\
\hline Perspective & 12 & 68 \\
\hline Exploration & 13 & 67 \\
\hline Impact & 13 & 67 \\
\hline Strategy & 18 & 67 \\
\hline Systems & 14 & 57 \\
\hline Framework & 11 & 54 \\
\hline Knowledge management & 10 & 50 \\
\hline Technology & 9 & 50 \\
\hline Strategic change & 9 & 49 \\
\hline
\end{tabular}

Fuente: Elaboración propia con base en VOSviewer

El programa VOSviewer con un umbral de $\geq 5$ co-ocurrencias por documento arrojo 57 palabras claves de las cuales solo se muestran las 20 más importantes en la tabla 3, observando que la que más citas tuvo fue aprendizaje organizacional con 107, seguido de cambio organizacional con 91, y si se agrega la palabra clave cambioorganizacional con 40 ocurrencias que está en el tercer lugar de palabras buscadas, sumaria un total de 131, con lo que sería el término con más ocurrencias; esto es lógico ya que fueron las palabras claves que se usaron en la búsqueda en Web of Science.
En lo que se refiere a la fuerza de enlace de las principales palabras claves del presente estudio, se puede determinar que el término aprendizaje organizacional tiene una fuerza de enlace de 372 , la palabra clave cambio organizacional tiene una fuerza de enlace de 288, en tanto que la palabra desempeño tuvo una fuerza de enlace de 200.

Por otra parte, los mapas de red sirven para interpretar gráficamente cuáles son los temas de investigación, ya que se muestran mediante etiquetas las palabras claves de más co-ocurrencia; el tamaño de la etiqueta es un indicativo de la importancia que tienen 
en el tema de investigación, y permiten efectuar un análisis más minucioso (van Eck \& Waltman, 2010; Fergnani, 2019).

En el siguiente mapa bibliométrico, se muestran los grupos generados por la base de datos Web of Science, los cuales se procesaron con el software del VOSviewer siendo las palabras claves utilizadas para la búsqueda: "organizational change" and "organizational learning", el mapa bibliométrico que se generó está conformado por 5 grupos, los que sirven de guía para conocer cómo se está estudiando el tema de investigación propuesto para este estudio.

Figura 4. Mapa de redes de palabras claves indexadas

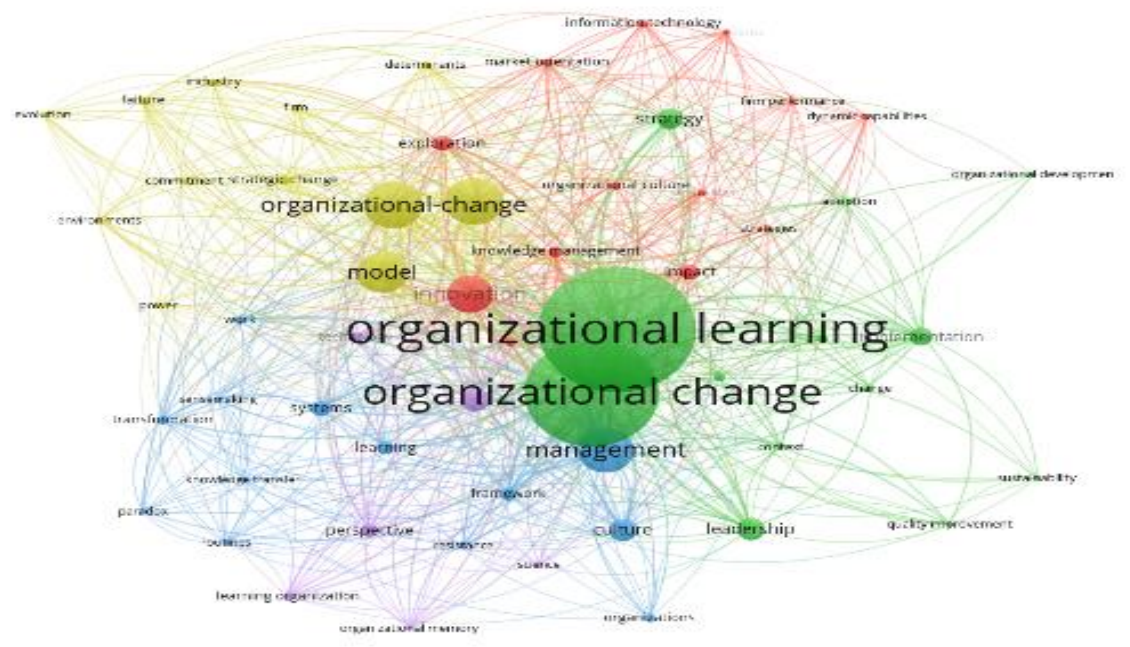

Fuente: software VOSviewer

En la figura 4, se observan las palabras claves con mayor co-ocurrencia ya que el VOSviewer las resalta con una etiqueta más grande que las demás, en este caso las que resaltan más son: "aprendizaje organizacional" (organizational learning) y "cambio organizacional" (organizational change) que como ya se mencionó fueron las palabras claves que se emplearon para esta investigación. De este modo en el mapa se aprecia cómo se agrupan las palabras, y se señalan con colores a que clúster pertenecen.

Por otro lado, el mapa de visualización de superposición muestra qué tópicos son los que se han trabajado a través del tiempo, y señala cuales se mantienen vigentes. Los años se simbolizan con distintos colores. Los temas que se han investigado en los últimos 5 a 8 años aparecen en colores rojo, naranja y amarillo, en tanto que las temáticas con más de 10 años son mostradas en colores verde y azul (Fergnani, 2019).

La figura 3 muestra otra vez la red de palabras claves; pero, con una perspectiva de línea del tiempo que refleja cómo ha evolucionado el estudio del cambio organizacional y el aprendizaje organizacional, los datos obtenidos de la base de datos WOS muestran resultados de investigaciones entre 2006 y 2012.

Figura 5. Mapa de visualización de superposición de las palabras claves 


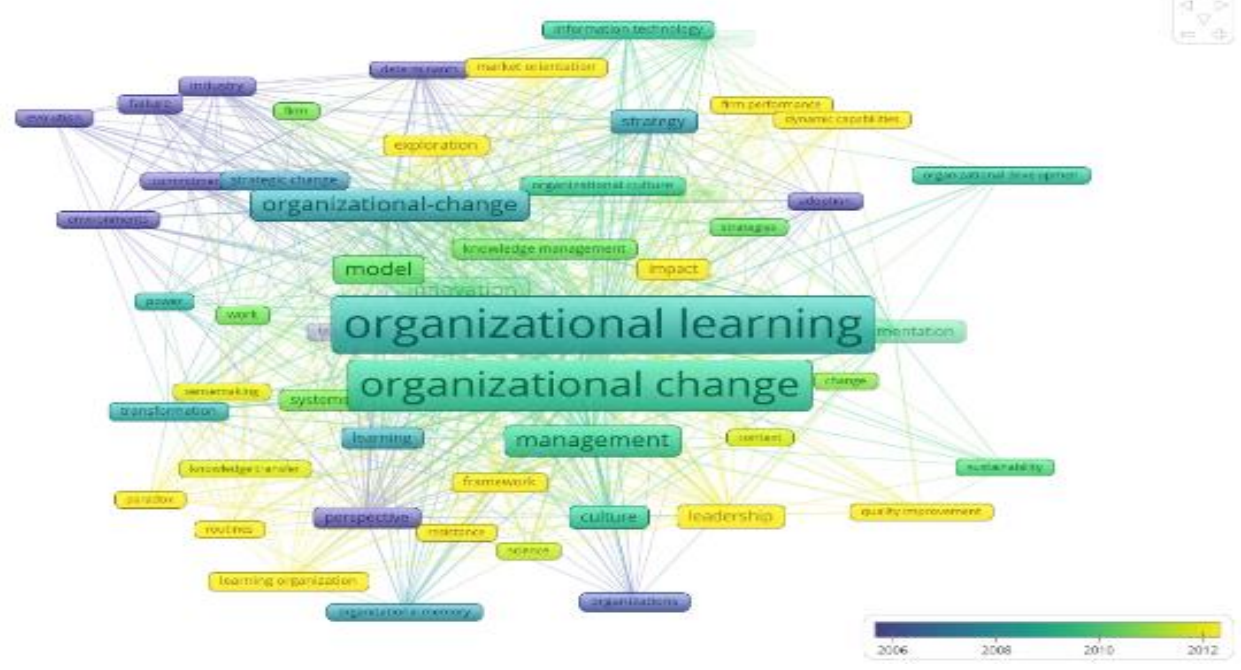

Fuente: software VOSviewer

Algunos de los temas de estudio que se muestran en la figura 5 que se encuentran en tendencia en años recientes son: organización que aprende, rutinas, paradoja, exploración, orientación al mercado, impacto, calidad, capacidades dinámicas, liderazgo, marco de referencia y mejoramiento de la calidad. Por su parte el cambio organizacional y el aprendizaje organizacional son temas que llevan más tiempo estudiándose, pero que se siguen estudiando en la actualidad, mientras que en el caso del término "organización", su estudio lleva más tiempo.
En el mapa de densidad, el programa VOSviewer muestra la intensidad de trabajo que tiene cada una de las palabras claves según el número de veces que aparecieron en los documentos consultados. Los colores indican cuales son las palabras claves más empleadas, en rojo se muestran las que tienen mayor intensidad y en azul las de menor cantidad de investigación, el exponer la información de esta forma permite ver claramente cuáles son los temas de mayor importancia en el mapa (Fergnani, 2019; Van Eck y Waltman, 2010).

Figura 6. Mapa de densidad de las palabras claves

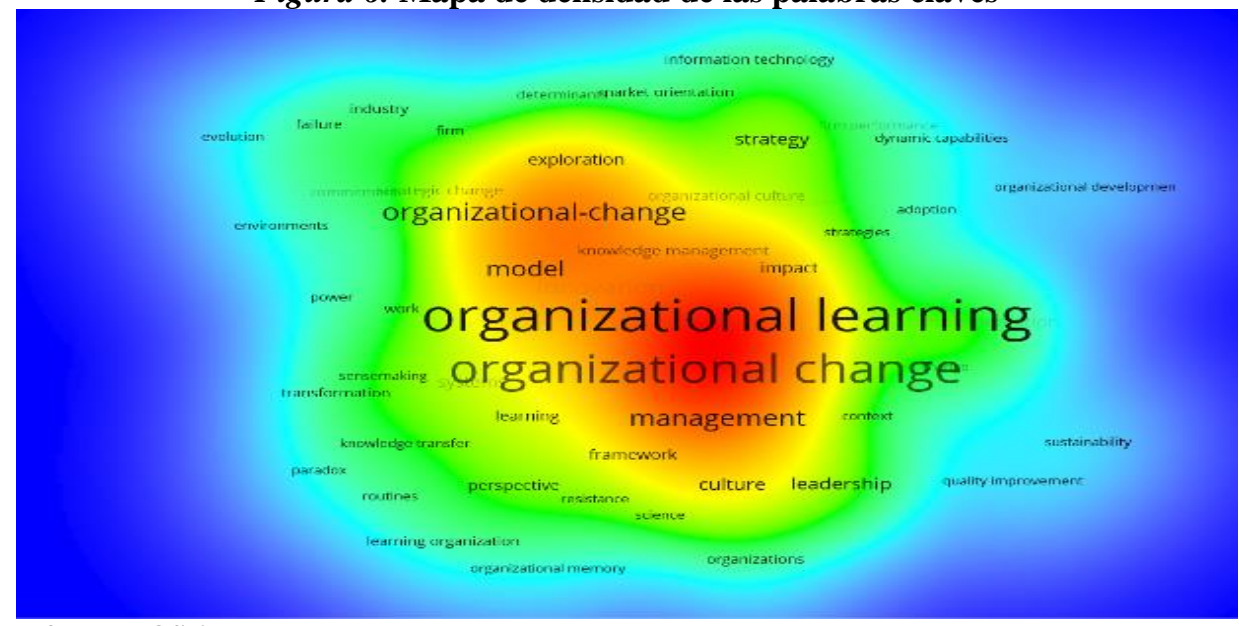

Fuente: software VOSviewer 
La figura 6 muestra el mapa de densidad de las palabras claves que tienen más relevancia en función del número de publicaciones en las que aparecen, en el cual, al igual que en el mapa de visualización de red se aprecia que las más relevantes son aprendizaje organizacional y cambio organizacional, seguidas de la palabra cambio-organizacional y administración.

\section{Clústeres}

El software del VOSviewer también clasifica a los grupos de palabras claves mostradas en los mapas bibliométricos y los denomina clúster, en la figura 7 se muestran los clústeres con distintos colores, ya que a cada grupo de palabras claves que conforman un clúster les asigna un color.

Un grupo o clúster es una serie de elementos que pertenecen a un mapa, y cuyo rasgo es que no pueden ser parte de distintos clústeres al mismo tiempo, las palabras claves que hay en un clúster determinan que es un atributo y estos son enumerados según los conglomerados que surjan del mapa de densidad. (Van Eck y Waltman, 2018).

Los clústeres se concentran de tal forma que las palabras claves que las incluyen sea más probable que las citen en un mismo documento o artículo, ya que se identifican los distintos clústeres, se determina cuál es la línea de estudio que siguen según las palabras claves que lo integran debido a que estos se pueden interpretar como macro-áreas de investigación, por lo que se les denominan según el campo de estudio al que correspondan las palabras claves de mayor importancia dentro del grupo. (Skute et al., 2019).

De este modo, los clústeres se generaron de acuerdo a los resultados de la base de datos de Web of Science y que fueron procesados en el programa del VOSviewer, siendo las palabras de búsqueda utilizadas: "organizational change" AND "organizational learning", el mapa bibliométrico creó 5 clústers, los que sirven como pauta para saber cómo se estudia el objeto de investigación propuesto para este trabajo de investigación.
Clúster 1. Este clúster cuenta con 13 ítems donde las palabras claves indexadas con mayor peso fueron: administración del conocimiento, organizaciones que aprenden, habilidades, orientación al mercado, estrategias, cultura organizacional. Por lo que se puede determinar que esta línea de investigación se centra en estudiar el manejo de las Organizaciones.

Clúster 2. Los trabajos de este clúster cuentan con 13 ítems, y las palabras claves indexadas más relevantes son: adopción, cambio, gestión del cambio, cambio organizacional, mejoramiento de la calidad, estrategia. Siendo la Gestión del Cambio Organizacional la tendencia en este clúster.

Clúster 3. Este clúster tiene 13 ítems de los cuales las palabras claves indexadas más importantes son: cultura, aprendizaje, administración, organizaciones, resistencia, sistemas, trabajo, entre otros. Las tendencias que se investigan en este clúster son las relacionadas con la Cultura organizacional.

Clúster 4. Este clúster cuenta con 12 ítems donde las palabras claves indexadas con mayor peso fueron: ambientes, empresa, industria, modelo, cambio-organizacional, desempeño, compromiso, cambio estratégico. Por lo que se puede determinar que esta línea de investigación se centra en el Entorno industrial.

Clúster 5. Los trabajos de este clúster cuentan con 6 ítems donde las palabras claves indexadas más relevantes fueron: conocimiento, organización que aprende, memoria organizacional, perspectiva, ciencia, tecnología. Por lo que las tendencias que se investigan en este clúster son las relacionadas con el Conocimiento.

\section{CONCLUSIONES}

Este trabajo tenía como finalidad: conocer cuál ha sido el desarrollo y avance que ha tenido el estudio de la relación que hay entre el cambio organizacional y el aprendizaje organizacional, por la influencia que tienen las organizaciones que aprenden en el manejo de las empresas. Por otra parte, también es importante saber de qué manera se ha abordado este tema dentro del ámbito académico, para así 
contar con una mejor perspectiva de qué líneas de investigación se tienen que impulsar. Ciertamente, es un tema de estudio bastante investigado, pero se puede concluir que, gracias a los incesantes cambios que hay en el mundo y en los mercados, se hace necesario seguir estudiándolo.

Los datos obtenidos de la consulta en la base de datos Web of Science indican que hay 217 documentos relacionados al tema.

Indudablemente la base de datos Web of Science es de la más importantes que hay, ya que proporciona información muy amplia, y muestra como este tema de estudio se empezó a estudiar cuando menos desde 1997, y se ha incrementado paulatinamente su estudio, sobre todo desde 2018, teniendo su punto máximo en el 2020.

En lo que se refiere a los países con mayor producción científica según la base de datos Web of Science, son EUA, que lidera la producción científica sobre la relación entre el cambio organizacional y el aprendizaje organizacional, le siguen muy lejos Inglaterra, Australia, Canadá, Francia y Alemania, que son países desarrollados y cuyas empresas sobresalen a nivel mundial.

En cuanto a los principales artículos, según el número de veces que fueron citados. Se tiene que el artículo más citado es "Organizational Learning: The Contributing Processes And The Literatures" con 3558 citas, publicado en la revista Organization Science en 1991, escrito por George P. Huber; el segundo artículo más citado es: "Capabilities, cognition, and inertia: Evidence from digital imaging" con 1107 citas, de Tripsas y Gavetti, del año 2000 publicado en la revista Strategic Management Journal, y el tercer artículo más citado, con 474 citas es de los autores Zollo \& Singh, publicado en el 2004 en la revista Strategic Management Journal titulado: "Deliberate learning in corporate acquisitions: Post-acquisition strategies and integration capability in US bank mergers".

En lo referente al área de estudio que ha mostrado mayor interés por abordar esta temática ha sido la de Administración (Management); desde la variedad de sus subáreas, es lógico ya que se busca comprender como el cambio organizacional y el aprendizaje organizacional son abordados desde la perspectiva de la administración y gestión empresarial y de negocios. Le siguen el área de Business, y luego el de Information Science Libraryscience.

Por otra parte, la información exhibida mediante las gráficas del programa del VOSviewer sirvió para poder ver los tópicos, así como los trabajos más relevantes por su contenido e impacto incluidos en el repositorio académico Web of Science sobre aprendizaje organizacional y cambio organizacional.

En cuanto al estudio de la relación entre el cambio organizacional y el aprendizaje organizacional hay 4 clústeres de investigación; en el primero de ellos están: Australia, Alemania, Inglaterra, y Suecia; el segundo clúster lo componen: Francia, Holanda, España y EUA, el tercer clúster está conformado por Canadá e Italia; y en el cuarto clúster se hallan Dinamarca y Noruega. Siendo los Estados Unidos el país en el que se generan las aproximaciones teóricas más ricas en los registros bibliométricos de la base de datos Web of Science. (Ver figura 3).

Por otra parte, se hizo un análisis y procedimiento de recuento de coocurrencia tomando como base las palabras claves indexadas, eligiendo un número mínimo de co-ocurrencias en las palabras claves $\geq 5$, la elección de este umbral se dio ya que el resultado de co-ocurrencias fue de 1003 palabras claves.

Las palabras que más citaciones tuvieron fueron: aprendizaje organizacional con 107 ocurrencias, seguido de cambio organizacional con $91 \mathrm{y}$ cambio-organizacional con 40. (Ver Tabla 3).

Las palabras claves con mayor coocurrencia son: "aprendizaje organizacional" (organizational learning) y "cambio organizacional" (organizational 
change) que fueron las palabras claves que se emplearon para esta investigación. (Ver Figura 4).

Como ya se indicó, el objetivo de esta investigación bibliométrica fue hacer un estudio que refleje las tendencias de investigación sobre la relación entre el cambio organizacional y el aprendizaje organizacional, para ello, la figura 5 muestra las variables que los trabajos publicados en las fechas más recientes arrojaron; dando como resultado las siguientes variables: organización que aprende, rutinas, paradoja, exploración, orientación al mercado, impacto, calidad, capacidades dinámicas, liderazgo, marco de referencia y mejoramiento de la calidad.

Por su parte, la figura 6 contiene el mapa de densidad que muestra que las palabras clave más relevantes son aprendizaje organizacional y cambio organizacional, seguidas de la palabra cambio-organizacional.

Por otra parte, el análisis efectuado con el software VOSviewer mostró las líneas de investigación más recurrentes a través de 5 clústeres que agrupan palabras claves que contienen los títulos y resúmenes de las bases de datos. Los 5 clústeres son:

El Clúster 1 cuenta con 13 ítems y su línea de investigación se centra en estudiar el Manejo de las Organizaciones.
El Clúster 2 tiene 13 ítems, siendo la Gestión del Cambio Organizacional la tendencia de estudio en este clúster.

El Clúster 3 abarca 13 ítems, y las tendencias que investiga este clúster se relacionan con la Cultura organizacional.

El Clúster 4 cuenta con 12 ítems, siendo su línea de investigación centrada en el Entorno industrial.

En el Clúster 5 hay 6 ítems, y se investiga el Conocimiento.

Finalmente, de acuerdo con la visión de la corriente de análisis del aprendizaje organizacional, las firmas empresariales que pretenden destacar sobre las demás, ser líderes y tener mayor competitividad; es importante que tengan la habilidad para cambiar, ya que no es algo que se pueda comprar, sino que resulta del aprendizaje que la propia empresa logra por su cuenta.

Por último, aunque este trabajo es una aproximación para tener una perspectiva de cómo se está estudiando la relación entre el cambio organizacional y el aprendizaje organizacional en la investigación científica; se sugiere efectuar en un futuro un análisis más a fondo de los clústeres que se hallaron en la presente investigación, y hacer un análisis del texto completo de los artículos más citados, para así comprender mejor este tema de investigación.

\section{REFERENCIAS}

Al Kasasbeh, M. M., Al Kasasbeh, S. A. M., \& AL Faouri, A. H. (2016). Smart organization characteristics and its impact on social and environmental performance: An empirical study on Jordan Phosphate Mines Company. International Journal of Business and Management, 11(8), 106-115.

Argyris, C. (2010). Organizational traps: Leadership, culture, organizational design. Oxford University Press.

Bahrami, M. A., Kiani, M. H., Montazeralfaraj, R., Zadeh, H. F., \& Zadeh, M. M. (2016). The mediating role of organizational learning in the relationship of organizational intelligence and organizational agility. Osong Public Health Research Perspect, 7(3), 190-196.

Bar-Ilan, J. (2008). Informetrics at the beginning of the 21 st century-A review. Journal of Informetrics, 2(1), 1-52.

Coghlan, D. (2013). Action Learning Research? Reflections from the Colloquium at the Third International Conference on Action Learning. Action Learning: Research and Practice, 10(1), 54-57

Cunliffe, A. L., \& Easterby-Smith, M. (2004). From reflection to practical reflexivity: experiential learning as lived experience. En Reynolds M. (Ed.), Organizing reflection. (30-46). 
Routledge.

Erçetin, Ş. Ş., \& Kayman, E. A. (2014). How to be a quantum leader in an intelligent organization? En Banerjee, S., \& Erçetin, Ş. Ş. (Eds.) Chaos, Complexity and Leadership 2012. (247252). Springer, Dordrecht.

Fergnani, A. (2019). Mapping futures studies scholarship from 1968 to present: A bibliometric review of thematic clusters, research trends, and research gaps. Futures, 105(2019), 104123.

Fernandez, S., \& Rainey, H. G. (2006). Managing successful organizational change in the public sector. Public Administration Review, 66(2), 168-176.

Fernandez, S., \& Pitts, D. W. (2007). Under what conditions do public managers favor and pursue organizational change? The American Review of Public Administration, 37(3), 324-341.

Gálvez, C. (2016). Visualización de las principales líneas de investigación en salud pública: Un análisis basado en mapas bibliométricos aplicados a la revista española de salud pública (2006-2015). Revista Española de Salud Pública, 90(1), 1-10.

Holba, A.; Bahr, P.T.; Birx D.L. \& Fischler M.J. (2019), Integral Learning and Working: Becoming a Learning Organization. New Directions for Higher Education, 185(185), 85-99.

Huber, G.P. (1991). Organizational Learning: The Contributing Processes And The Literatures. Organization Science, 2(1),88-115.

James, E., \& Alana, E. (2018). Action Learning for Higher Ed Technology: Learning What Supports Graduate Students. Organization Development Journal, 36(4), 17-29.

Keskin, H., \& Balak, D. (2020). Smart Organizations from Organizational Intelligence Perspective. Business and Economics Research Journal; Bursa, 11(4): 1083-1096.

Kulkarni, A. V., Aziz, B., Shams, I., \& Busse, J. W. (2009). Comparisons of citations in Web of Science, Scopus, and Google Scholar for articles published in general medical journals. JAMA, 302(10), 1092-1096.

Liebowitz, J. (2000). Building organizational intelligence: A knowledge management primer. CRC Press.

Limwichitr, S., Broady-Preston, J., \& Ellis, D. (2015), A discussion of problems in implementing organisational cultural change: Developing a learning organisation in University Libraries, Library Review, 64(6/7), 480-488.

London, M., \& Sessa, V. I. (2006). Continuous learning in organizations: A living systems analysis of individual, group, and organization learning. Research in Multi-Level Issues. Emerald Group Publishing Limited, Bingley, 5(1), 123-172.

Marquardt, M. (2000). Action learning and leadership. The Learning Organization, 7(5), 233-241.

Olczyk, M. (2016). A systematic retrieval of international competitiveness literature: A bibliometric study. Eurasian Economic Review, 6(3), 429-457.

Pole, J. G., Madsen, E., \& Dishman, P. (2000). Competitive intelligence as a construct for organizational change. Competitive Intelligence Review, 11(4), 25-31.

Rahdarpour, J., \& Sheykhi, A. (2016). Relationship between organizational intelligence, organizational learning, intellectual capital and social capital using SEM. International Journal of Humanities and Cultural Studies, 2(4), 2076-2086.

Ravichandran, N., \& Mishra, R. (2018). Toward building HR competencies: A shift from the nonlearning toward the learning organization. International Journal of Healthcare Management, 11(3), 233 - 238.

Revans, R. W. (1998). The ABC of action learning. Lemos and Crane.

Rigg, C., \& Trehan, K. (2004). Reflections on working with critical action learning. Action Learning: Research and Practice, 1(2), 149-165.

Salge, T. O. \& Vera, A. (2012), Benefiting from Public Sector Innovation: The Moderating Role of Customer and Learning Orientation. Public Administration Review, 72(4), 550-559.

Senge, P. M. (2006). The fifth discipline: the art and practice of the learning organization. Doubleday/ Currency 
Schwaninger, M. (2019). Governance for intelligent organizations: A cybernetic contribution. Kybernetes, 48(1), 35-57.

Skute, I., Zalewska-Kurek, K., Hatak, I., y de Weerd-Nederhof, P. (2019). Mapping the field: A bibliometric analysis of the literature on university-industry collaborations. The Journal of Technology Transfer, 44(3), 916-947.

Šneiderienè, A., Vaitiekus, A., y Vaitiekienè, J. (2020). Opportunities for Leadership Expression in a Learning Organization. Socialiniai Tyrimai, 43(1), 45-57.

Tortorella, G. L. \& Cawley-Vergara, A. M., Garza-Reyes, J. A. \& Sawhney, R. (2020). Organizational learning paths based upon industry 4.0 adoption: An empirical study with Brazilian manufacturers, International Journal of Production Economics, 219(C), 284294.

Tripsas, M., \& Gavetti, G. (2000). Capabilities, cognition, and inertia: Evidence from digital imaging. Strategic Management Journal, 21(10-11), 1147-1161.

Ugoani, J.N. (2016). Emotional Intelligence And Organizational Competitiveness: Management Model Approach. Independent Journal of Management \& Production; Sao Paulo, 7(3): 786-806.

Van Eck, N. J., \& Waltman, L. (2010). Software survey: VOSviewer, a computer program for bibliometric mapping. Scientometrics, 84(2), 523-538.

Van Raan, A. (2019). Measuring Science: Basic Principles and Application of Advanced Bibliometrics. En Glänzel, W., Moed, H. F., Schmoch,U., \& Thelwall, M. (Eds.), Springer Handbook of Science and Technology Indicators. (237-280). Springer International Publishing.

Virany, B., Tushman, M. L., \& Romanelli, E. (1992). Executive succession and organization outcomes in turbulent environments: An organization learning approach. Organization Science, 3(1), 72-91.

Walker, C. (2014). Organizational learning: the role of third party auditors in building compliance and enforcement capability. International Journal of Auditing, 18(3), 213-222.

Zollo M. \& Singh, H. (2004). Deliberate learning in corporate acquisitions: Post-acquisition strategies and integration capability in US bank mergers, Journal Strategic Management, 25(13), 1233-1256. 